\title{
Bacterial Etiology of Otitis Media and their Antibiotic Susceptibility Pattern among Patients coming to a Tertiary Care Hospital, Jaipur, India
}

\author{
${ }^{1}$ Mohit Agrawal, ${ }^{2}$ Neha Gupta, ${ }^{3}$ Gunjan Pandey
}

\begin{abstract}
Aim: The aim of the study was to identify the microorganisms associated with the chronic discharging ears and their antimicrobial susceptibility pattern.

Materials and methods: A total of 251 samples of ear swabs were received at Mahatma Gandhi Medical College \& Hospital, Jaipur, Rajasthan, India. Pus samples were taken from external auditory canal using sterile cotton swabs and then cultured on different microbiological media. Antibiotic sensitivity was tested and interpreted by method according to Clinical and Laboratory Standards Institute standards.

Results: Out of 251 samples received, 135 (53.78\%) samples had growth. The microbiology of these 135 samples was studied. Pseudomonas aeruginosa was the major organism isolated from 88 samples followed by Staphylococcus aureus (37), and other coliforms (10). Drug susceptibility of $P$. aeruginosa showed that colistin was active against all isolates followed by piperacillin-tazobactam and imipenem. Approximately $60 \%$ S. aureus isolates were methicillin-resistant S. aureus. Vancomycin, linezolid, and teicoplanin were $100 \%$ sensitive.

Conclusion: From the current study, it was concluded that $P$. aeruginosa is the potential cause of ear-associated infections. All isolates of $P$. aeruginosa were susceptible to colistin, and $S$. aureus was susceptible to vancomycin, linezolid, and teicoplanin.
\end{abstract}

Keywords: Chronic suppurative otitis media, methicillin-resistant S. aureus, Pseudomonas aeruginosa, Staphylococcus aureus,

How to cite this article: Agrawal M, Gupta N, Pandey G. Bacterial Etiology of Otitis Media and their Antibiotic Susceptibility Pattern among Patients coming to a Tertiary Care Hospital, Jaipur, India. J Mahatma Gandhi Univ Med Sci Tech 2017;2(1):7-9.

Source of support: Nil

Conflict of interest: None

\section{INTRODUCTION}

Otitis is a general term for inflammation or an infection of the human ear. It is divided as otitis externa, which

\footnotetext{
${ }^{1}$ Assistant Professor, ${ }^{2}$ Resident, ${ }^{3}$ Senior Demonstrator

${ }^{1-3}$ Department of Microbiology, Mahatma Gandhi Medical College \& Hospital, Jaipur, Rajasthan, India
}

Corresponding Author: Mohit Agrawal, Assistant Professor Department of Microbiology, Mahatma Gandhi Medical College \& Hospital, Jaipur, Rajasthan, India, e-mail: mohit_wal@yahoo.com involves outer ear and ear canals. Otitis media and otitis interna are involvements of middle ear and inner ear respectively.

Children tend to have higher predisposition to ear infection than adults because anatomy of the Eustachian tube in children permits easier access of organism through the nasopharynx. ${ }^{1}$

Otitis media is a major health problem and occurs with a high incidence and prevalence in both developed and developing countries. ${ }^{2,3}$ It can be either suppurative or nonsuppurative. The inflammation often begins when infections that cause sore throat, cold, or other respiratory or breathing problems spread to middle ear. ${ }^{4}$ Otitis media could be of viral or bacterial origin. It could present an acute or chronic course. ${ }^{5}$ Acute otitis media (AOM) is a common childhood illness. An AOM episode, i.e., not diagnosed promptly or is inadequately treated can lead to chronic suppurative otitis media (CSOM). ${ }^{6}$ It is characterized by the presence of persistent perforation of tympanic membrane with recurrent or persistent mucoid/ mucopurulent discharge for at least 4 to 6 weeks. ${ }^{7-10}$ Persistence of purulent otorrhea may lead the mucous membrane to become ulcerated, polypoid, or granulomatous. Resorption of the ossicles and mastoid bone due to demineralization and osteoclastic activity may be seen in prolonged infection.

Around 30 million cases of CSOM occur worldwide every year, with a large number in pediatric population.

The organisms isolated in chronic otitis media can be aerobes, anaerobes, mixed, or fungi. Various studies done in United States, United Kingdom, Japan, Ghana, India revealed that the common causative organism may be aerobic (e.g., Pseudomonas aeruginosa, Escherichia coli, Staphylococcus aureus, Streptococcus pyogenes, Proteus mirabilis, Klebsiella species) or anaerobic (e.g., Bacteroides, Peptostreptococcus, etc.) or mixed infections / organisms. ${ }^{7,-11}$

In discharging ear, both topical and systemic therapy are employed for treatment. The systemic therapy is chosen depending on the organism isolated in culture.

Our study is aimed to determine the prevalence and pattern of bacteria isolates and their antimicrobial susceptibility pattern in cases of CSOM. 


\section{MATERIALS AND METHODS}

The study was carried out from January 1, 2016, to June 30, 2016, in Microbiology Department at Mahatma Gandhi Medical College \& Hospital, Jaipur, Rajasthan, India. The study was conducted on relevant specimens received in microbiology department lab. These specimens were obtained from adults and children of both sexes coming to the ENT department with history of fever, ear pain, and ear discharge.

Single-use commercially available sterile cotton swabs were used to collect the pus, by inserting and rotating the swab, as deep as possible in ear, without hurting the patient. All samples were collected by standard microbiological technique, and outmost care was taken to avoid contamination.

The pus swabs were cultured on sheep blood agar, chocolate agar, and MacConkey's agar and incubated at $37^{\circ} \mathrm{C}$ for 24 to 48 hours. The blood agar and chocolate agar plates were incubated at $5 \% \mathrm{CO}_{2}$ at $37^{\circ} \mathrm{C}$. After overnight incubation, suspected colonies of isolated organism were identified according to the standard microbiological methods (colony morphology, hemolysis, metallic sheen, pigmentation, and Gram stain). Antibiotic susceptibility testing was performed on Mueller Hinton agar as per Clinical and Laboratory Standards Institute (CLSI) standards. ${ }^{12}$

Using Kirby-Bauer disk diffusion method, the following antibiotics were tested for susceptibility testing of Gram-negative organisms:

Amikacin $(30 \mu \mathrm{g})$, Ampicillin $(10 \mu \mathrm{g})$, AmoxyClavulanate $(20 / 10 \mu \mathrm{g})$, Aztreonam (30 $\mu \mathrm{g})$, Cefepime

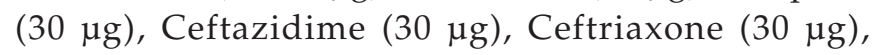
Ciprofloxacin $(5 \mu \mathrm{g})$, Colistin $(10 \mu \mathrm{g})$, Gentamycin $(30$ $\mu \mathrm{g})$, Imipenem $(10 \mu \mathrm{g})$, Levofloxacin $(5 \mu \mathrm{g})$, Meropenem $(10 \mu \mathrm{g})$, Piperacillin-tazobactam $(100 / 10 \mu \mathrm{g})$, and Ticarcillin-clavulanate $(75 / 10 \mu \mathrm{g})$.

The following antibiotics were tested for susceptibility testing of Gram-positive organisms:

Amikacin $(30 \mu \mathrm{g})$, Azithromycin $(15 \mu \mathrm{g})$, Cefoxitin (30 $\mu \mathrm{g})$, Clindamycin $(2 \mu \mathrm{g})$, Cotrimoxazole $(1.25 / 23.75 \mu \mathrm{g})$, Doxycycline $(30 \mu \mathrm{g})$, Erythromycin $(15 \mu \mathrm{g})$, Gentamycin $(10 \mu \mathrm{g})$, Linezolid (30 $\mu \mathrm{g})$, Penicillin G (10U), Teicoplanin (30 $\mu \mathrm{g})$, and Vancomycin $(30 \mu \mathrm{g})$.

Zone of inhibition around each antibiotic disk was interpreted with the help of CLSI standards M100 S25.

\section{RESULTS}

A total of 251 ear swab samples were received in study duration. Of these, 135 samples showed growth on culture, giving isolation rate of $53.78 \%$. The most common organism isolated was P. aeruginosa 88 (65.18\%) followed by S. aureus $37(27.4 \%)$. Coliforms were isolated in 10 (7.4\%) patients (Tables 1 and 2 ).
Table 1: Drugs susceptibility to $P$. aeruginosa

\begin{tabular}{ll}
\hline Antibiotic & $n$ (susceptibility \%) \\
\hline Amikacin & $47(53.41 \%)$ \\
Cefepime & $41(46.59 \%)$ \\
Ceftazidime & $46(52.27 \%)$ \\
Ciprofloxacin & $44(50 \%)$ \\
Colistin & $88(100 \%)$ \\
Gentamycin & $45(51.13 \%)$ \\
Imipenem & $49(55.68 \%)$ \\
Piperacillin-tazobactam & $56(63.63 \%)$ \\
\hline
\end{tabular}

Table 2: Drugs susceptibility to $S$. aureus

\begin{tabular}{ll}
\hline Antibiotic & $n$ (susceptibility \%) \\
\hline Amikacin & $35(94.59 \%)$ \\
Cefoxitin & $15(40.54 \%)$ \\
Clindamycin & $19(51.35 \%)$ \\
Erythromycin & $08(21.62 \%)$ \\
Gentamycin & $32(86.48 \%)$ \\
Linezolid & $37(100 \%)$ \\
Teicoplanin & $37(100 \%)$ \\
Vancomycin & $37(100 \%)$ \\
\hline
\end{tabular}

\section{DISCUSSION}

Otitis media is a serious health care concern worldwide. Previous publications have reported its incidence to depend on socioeconomic factors, like poor living conditions, over-crowding, poor hygiene, and nutrition as basis for the widespread of the CSOM in developing countries. $^{13}$

In our study, the pattern of bacterial isolates showed that $P$. aeruginosa $(65.18 \%$ ) was the most prevalent bacterial organism found in the middle ear with CSOM, which is similar to the finding elsewhere. ${ }^{13-15}$ This is followed by $S$. aureus (27.4\%) as the second most common, which also corresponds to other studies in other centres. ${ }^{11,14}$ Coliforms (i.e., E. coli, P. mirabilis) were relatively low $(7.40 \%)$. Anaerobic culture was not carried out and no fungi was isolated in our samples.

Antimicrobial susceptibility of $P$. aeruginosa in our study revealed that all the isolates were susceptible for colistin. Piperacillin-tazobactam (63.63\%) and Imipenem $(55.68 \%)$ were next two most sensitive antibiotics. Antipseudomonal cephalosporins did not fare well and were less sensitive in comparison to amikacin (53.41\%).

Among isolated S. aureus, approximately $60 \%$ were methicillin-resistant S. aureus (MRSA). Vancomycin, linezolid, and teicoplanin were $100 \%$ sensitive. Aminoglycosides, amikacin (94.59\%), and gentamycin (86.48\%) were sensitive in most of the isolates. Susceptibility testing of coliforms was also carried out. 


\section{CONCLUSION}

Pseudomonas aeruginosa is a major cause of ear infections. Empirical antibiotic coverage is needed to cover this pathogen as it develops resistance very rapidly. Empirical therapy should be revised after result of culture and sensitivity so that targeted therapy is given to eradicate the pathogen. In our study, P. aeruginosa was the most common isolate followed by $S$. aureus. Pseudomonas aeruginosa showed good susceptibility to colistin, piperacillin-tazobactam, and imipenem. Majority of S. aureus were MRSA and sensitive to vancomycin, linezolid, and teicoplanin.

\section{REFERENCES}

1. Anthony W. Anatomy and ultrastructure of the human ear. In: Band BJ, Kerr AG, editors. Scott-Brown's otolaryngology, Basic science. 6th ed. London: Butterworth-Heinemann; 1997. p. $1 / 1 / 7-1 / 1 / 26$.

2. EI-Gendy GD. The incidence of otitis media with effusion in Menoufiya school children [MD thesis]. Faculty of Medicine, Menoufiya University; 1998.

3. St Sauver J, Marrs CF, Foxman B, Somsel P, Madera R, Gilsdorf JR. Risk factors for otitis media and carriage of multiple strains of Haemophilus influenza and Streptococcus pneumonia. Emerg Infect Dis 2000 Nov-Dec;6(6):622-630.

4. Berman S, Byrns PJ, Bondy J, Smith PJ, Lezotte D. Otitis media-related antibiotic prescribing patterns, outcomes and expenditures in a pediatric medicaid population. Pediatrics 1997 Oct;100(4):585-592.
5. Farooqui B, Jillani SA, Khursheed M. Spectrum of organisms associated with suppurative otitis media and their antibiotic sensitivity pattern. Pak J Otolaryngol 1990;6:99-104.

6. Leach AJ, Morris PS. Antibiotics for the prevention of acute and chronic suppurative otitis media in children. Cochrane Database Syst Rev 2006 Oct 18;(4):CD004401.

7. Tahira M. Mohammed AM, Gulnaz K, Mustafa K. Pseudomonas aeruginosa in chronic suppurative otitis media: sensitivity spectrum against various antibiotics in Karachi. J Ayub Med Coll Abbottabad 2009 Apr-Jun;21(2):120-123.

8. Berman S. Otitis media in children. N Eng J Med 1995;332(23): 1560-1565.

9. Yeo SG, Park DC, Hong SM, Cha CI, Kim MG. Bacteriology of chronic suppurative otitis media - a multicentre study. Acta Otolaryngol 2007 Oct;127(10):1062-1067.

10. Ibekwe AO. Chronic suppurative otitis media in Nigerian children. J Paediatr 1985;12:17-19.

11. Oni AA, Bakare RA, Nwaorgu OG, Ogunkunle MO, Toki RA. Bacterial agents of discharging ears and antimicrobial sensitivity pattern in children in Ibadan, Nigeria. West Afr J Med 2001 Apr-Jun;20(2):131-135.

12. CLSI Performance standards for antimicrobial disk susceptibility test: 2015. 25th Informational Supplement, M100-S25. Wayne (PA) :CLSI. p. 1-236.

13. Ologe FE, Nwawolo CC. Prevalence of chronic suppurative otitis media (CSOM) among school children in a rural community in Nigeria. Niger Postgrad Med J 2002 Jun;9(2):63-66.

14. Brook I, Frazier E. Microbial dynamics of persistent purulent otitis media in children. J Pediatr 1996 Feb;128(2):237-240.

15. Bluestone CD. Pathogenesis and epidemiology of chronic otitis media. WHO/CIBA Foundation Workshop, Report on prevention of hearing impairment for chronic otitis media held at CIBA foundation, London; 1996. p. 14-17. 